\title{
Is transdermal menopausal hormone therapy a safer option than oral therapy?
}

\author{
Bette Liu MBBS DPhil
}

See related research article by Racine and colleagues on page 555 and at www.cmaj.ca/lookup/doi/10.1503/cmaj.121490

$\mathrm{L}$ arge trials and observational studies have provided much-needed evidence on the benefits and harms of menopausal hormone therapy. Most of this evidence comes from studies involving women using oral formulations of estrogen alone or combined with a progestin. Established benefits of menopausal hormone therapy include the relief of menopausal symptoms and fracture prevention. For certain formulations, the known adverse effects include an increased risk of breast, ovarian and endometrial cancer, venous thrombosis and gallbladder disease. In their CMAJ article, Racine and colleagues report on the rates of cholecystectomy in a study involving more than 70000 women in France, where transdermal menopausal hormone therapy is much more commonly prescribed than oral preparations. ${ }^{1}$

In their observational prospective study, Racine and colleagues obtained data on menopausal hormone therapy and cholecystectomy from questionnaires completed by the participants every 2 years. The type of hormone therapy used was updated in analyses throughout the follow-up period. Over a mean of 11.5 years, 2819 women reported having had a cholecystectomy. The authors found that, compared with women who never used menopausal hormone therapy, women using oral estrogen regimens had an increased risk of cholecystectomy; the risk was not increased among those using transdermal regimens.

Only one previous study had sufficient statistical power to compare transdermal and oral routes of menopausal hormone therapy and the risk of gallbladder disease and cholecystectomy. The UK Million Women Study, a prospective observational cohort study, followed 1 million women for a mean of 6.1 years, during which 17190 women were admitted to hospital for cholecystectomy. ${ }^{2}$ Similar to the findings of Racine and colleagues, the risk of gallbladder disease and cholecystectomy was significantly lower among women using transdermal hormone therapy than among those using oral therapy (relative risk [RR] 1.17 v. $1.74 ; p<0.001)$ relative to women who never used menopausal hormone therapy. Regardless, the risks of gallbladder disease and cholecystectomy associated with transdermal therapy were still slightly elevated compared with women who never used menopausal hormone therapy (RR 1.17, 95\% confidence interval 1.10-1.24).

Transdermal menopausal hormonal therapy has primarily been designed to deliver exogenous estrogens directly to the systemic circulation. The addition of progestin, if considered necessary, is usually as an oral tablet, but some transdermal progestin delivery systems are available. Transdermal estrogen therapy is most commonly available as a patch or gel that is applied directly to the skin, with absorption of the active hormone into the systemic circulation. Patches have the advantage of needing to be changed only once or twice weekly, whereas gels usually need to be administered daily. As for disadvantages, patches can fall off, may cause skin irritation and can be unsightly; the use of a gel may result in inadvertent administration of estrogen to another person if skin-to-skin contact occurs before the gel has been fully absorbed.

In North America ${ }^{3}$ and other high-income countries such as the United Kingdom ${ }^{2}$ and the Netherlands, ${ }^{4}$ oral formulations of menopausal hormone therapy are much more commonly prescribed than transdermal ones. Why these differences exist is unclear, but there are some possible explanations. First, transdermal preparations are relatively new to the market, and physicians and patients may simply be more familiar and comfortable with the oral formulations. Second,
Competing interests: None declared.

This article was solicited and has not been peer reviewed.

Correspondence to: Bette Liu,

bette.liu@unsw.edu.au

CMAJ 2013. DOI:10.1503 /cmaj.130004 
transdermal preparations may be more costly in some settings. Third, because the prevailing practice has been to prescribe both estrogen and progestin for most women (among those who have not had a hysterectomy), the dosing regimen for transdermal therapy becomes more complex with the addition of a progestin pill. In the UK Million Women Study, transdermal formulations were more commonly used for estrogen-only hormone therapy, and consequently most women receiving transdermal therapy had had a hysterectomy. ${ }^{2}$

In France, unlike most other countries, most women prescribed menopausal hormone therapy are given a transdermal estrogen formulation with or without oral progestin. ${ }^{1}$ Therefore, the use of transdermal therapy in the study by Racine and colleagues was not strongly influenced by a woman's history of having a hysterectomy. Despite the prescribing differences between the UK and France, the results from both studies suggest that transdermal estrogen therapy is associated with a lower risk of cholecystectomy than oral estrogen therapy. This consistency across different populations gives further weight to the findings.

Other effects of menopausal hormonal therapy, such as a reduced risk of fracture and an increased risk of breast cancer, have been found not to differ between users of oral and transdermal preparations. ${ }^{5,6}$ However, there is increasing evidence that the use of transdermal menopausal hormone therapy confers no increase in the risk of venous thromboembolism, in contrast to the use of oral preparations, which are associated with an increased risk. ${ }^{7.8}$ Orally administered hormones are first metabolized by the liver before entering the systemic circulation, and metabolites are excreted in the bile and urine. Transdermally administered estrogen avoids this first-pass metabolism and can therefore be given in lower doses for equivalent physiologic effects. The different doses and concentrations of metabolites may explain the different effects observed with oral and transdermal routes of menopausal hormone therapy and gallbladder disease. ${ }^{2}$ For venous thromboembolism, an explanation for the different effects by route of hormone administra- tion is not as clear, although it has been suggested that the metabolites generated from the first-pass metabolism of estrogen may induce thrombogenic changes. ${ }^{7}$

Drug regulatory bodies currently recommend that hormone therapy be prescribed only for moderate to severe menopausal symptoms and that the dose and duration of use be minimized. ${ }^{9}$ The results from the study by Racine and colleagues support these recommendations. Unfortunately, there are no large clinical trials comparing transdermal and oral therapies, and such trials will probably never be conducted. Evidence to guide recommendations on the best route of hormone administration for individual women and prescribers will thus be limited to the growing volume of observational data. These data increasingly suggest that the overall risk-benefit profile of transdermal menopausal hormone therapy makes it a more attractive option than oral therapy.

\section{References}

1. Racine A, Bijon A, Fournier A, et al. Menopausal hormone therapy and risk of cholecystectomy: a prospective study based on the French E3N cohort. CMAJ 2013;185:555-61.

2. Liu B, Beral V, Balkwill A, et al.; Million Women Study Collaborators. Gallbladder disease and use of transdermal versus oral hormone replacement therapy in postmenopausal women: prospective cohort study. BMJ 2008;337:a386.

3. Hersch AL, Stefanik ML, Stafford RS. National use of postmenopausal hormone therapy: annual trends and response to recent evidence. JAMA 2004;291:47-53.

4. Faber A, Bouvy M, Loskamp L, et al. Dramatic change in prescribing of hormone replacement therapy in the Netherlands after publication of the Million Women Study: a follow-up study. Br J Clin Pharmacol 2005;60:641-7.

5. Beral V; Million Women Study Collaborators. Breast cancer and hormone-replacement therapy in the Million Women Study. Lancet 2003;362:419-27.

6. Banks E, Beral V, Reeves G, et al.; Million Women Study Collaborators. Fracture incidence in relation to the pattern of use of hormone therapy in postmenopausal women. JAMA 2004;291: 2212-20.

7. Canonico M, Oger E, Plu-Bureau G, et al. Hormone therapy and venous thromboembolism among postmenopausal women: impact of the route of estrogen administration and progestogens: the ESTHER Study. Circulation 2007;115:840-5.

8. Sweetland S, Beral V, Balkwill A, et al. Venous thromboembolism risk in relation to use of different types of postmenopausal hormone therapy in a large prospective study. J Thromb Haemost 2012; Sept. 10 [Epub ahead of print].

9. Banks E, Canfell K. Invited commentary: hormone therapy risks and benefits - the Women's Health Initiative findings and the postmenopausal estrogen timing hypothesis. Am J Epidemiol 2009; 170:24-8

Affiliation: Bette Liu is with the Faculty of Medicine, University of New South Wales, Sydney, Australia. 